\title{
Assessment of Various Density Functionals and Basis Sets for the Calculation of Molecular Anharmonic Force Fields
}

\author{
A. Daniel Boese \\ Institute of Nanotechnology, Forschungszentrum Karlsruhe, \\ P.O. Box 3640, D-76021 Karlsruhe, Germany \\ Wim Klopper \\ Lehrstuhl für Theoretische Chemie, Institut für Physikalische Chemie, \\ Universität Karlsruhe (TH), D-76128 Karlsruhe, Germany \\ Jan M. L. Martin* \\ Department of Organic Chemistry, Weizmann Institute of Science, IL-76100 Rehovot, Israel \\ (Dated: Received Feb. 14, 2005; Final form Mar. 15, 2005; Int. J. Quantum. Chem., in press)

\begin{abstract}
In a previous contribution (Mol. Phys. 103, xxxx, 2005), we established the suitability of density functional theory (DFT) for the calculation of molecular anharmonic force fields. In the present work, we have assessed a wide variety of basis sets and exchange-correlation functionals for harmonic and fundamental frequencies, equilibrium and ground-state rotational constants, and thermodynamic functions beyond the RRHO (rigid rotor-harmonic oscillator) approximation. The fairly good performance of double-zeta plus polarization basis sets for frequencies results from an error compensation between basis set incompleteness and the intrinsic error of exchange-correlation functionals. Triple-zeta plus polarization basis sets are recommended, with an additional highexponent $d$ function on second-row atoms. All conventional hybrid GGA functionals perform about equally well: high-exchange hybrid GGA and meta-GGA functionals designed for kinetics yield poor results, with the exception of of the very recently developed BMK functional which takes a middle position along with the $\mathrm{HCTH} / 407$ (second generation GGA) and TPSS (meta-GGA) functionals. MP2 performs similarly to these functionals but is inferior to hybrid GGAs such as B3LYP and B97-1.
\end{abstract}

*Electronic address: comartin@wicc.weizmann.ac.il 


\section{INTRODUCTION}

In recent years, Density Functional Theory (DFT) has perhaps been the most commonly applied method within the field of computational chemistry. Harmonic frequencies can routinely be computed, and assist experimentalists in the assignment of their measured infrared spectra.

Several contributions have been made in the last years which have established the computation of anharmonic force fields more firmly. The calculation of fundamental frequencies by the use of anharmonic force fields allows direct comparison between computed and observed spectroscopic transitions in contrast to harmonic frequencies, including resonances between several modes as well as overtones.

In the field of computational thermochemistry, quartic force fields provide both zeropoint vibrational energies (ZPVEs) and thermal corrections beyond the rigid rotor-harmonic oscillator approximation. In applications where high-temperature data are important, anharmonic effects will become non-negligible[1] and the computation of such corrections will become more important.

As early as 1997, DFT was employed for the computation of anharmonic force fields of polyatomic molecules. Thiel and coworkers studied three small molecules using several generalized gradient approximation (GGA) functionals 2]. In the following years, calculations of anharmonic force fields using DFT have been few and far between: Handy and coworkers have computed the force fields of ammonia [3], benzene [4] and diazomethane [5] with hybrid density functionals.

Starting in 2003, an increasing number of papers reporting DFT anharmonic force fields have been published and several groups have been working on this subject. Noteworthy are the activities in the Handy group, with studies of furan, pyrrole and thiophene [6] and phosphorus pentafluoride[7]. Barone[8] and two of us 9] have independently published two detailed studies on the azabenzene series; in the latter paper, we also explored the possibility of combining DFT anharmonic force fields with coupled cluster geometries and harmonic frequencies. As for validation studies, the group of Hess[10] has published one on a small number of mainly triatomic molecules. Barone and coworkers have published a series of articles [11, 12, 13, 14, 15] about the calculation of anharmonic force fields. Their validation set consists of three larger polyatomic molecules $\left(\mathrm{CH}_{2} \mathrm{NH}, \mathrm{H}_{2} \mathrm{CO}\right.$, and $\left.\mathrm{C}_{2} \mathrm{H}_{4}\right)$ plus two larger 
organic molecules (tetrazine and benzene) to compare harmonic frequencies.

Finally, we have published a detailed study on the calculation of quartic force fields [20], derived spectroscopic constants (vibrational anharmonicities, rotation-vibration coupling constants), and thermodynamic functions, all of which were compared to the corresponding data obtained from accurate ab initio (large basis set coupled cluster) quartic force fields. The validation set included 17 molecules, namely $\mathrm{C}_{2} \mathrm{H}_{2}$ 16], $\mathrm{C}_{2} \mathrm{H}_{4}[17], \mathrm{CCl}_{2}, \mathrm{CF}_{2}$ 18], $\mathrm{CH}_{2} \mathrm{NH}$ [19], $\mathrm{CH}_{2}$ [20], $\mathrm{CH}_{4}$ [21], $\mathrm{H}_{2} \mathrm{CO}$ 22], $\mathrm{H}_{2} \mathrm{O}[1], \mathrm{H}_{2} \mathrm{~S}[23], \mathrm{HCN}[20], \mathrm{N}_{2} \mathrm{O}[24], \mathrm{NH}_{2}$ [25], $\mathrm{PH}_{3}[26], \mathrm{SiF}_{4}[27], \mathrm{SiH}_{4}[28]$, and $\mathrm{SO}_{2}$ [29]: the citations accompanying each molecule point to the source for the $a b$ initio reference data. In that same paper, we also considered (for a subset of molecules) convergence in terms of the integration grids used for energy+gradient and for the CPKS (coupled perturbed Kohn-Sham) step, as well as in terms of the step size employed for the numerical differentiation. Finally, we assessed the performance of various GGA and hybrid GGA exchange-correlation functionals, specifically BLYP [30], HCTH407 [31], and PBE [32] among the former, and B3LYP 33], B97-1 34], B97-2 [35], and PBE0 36] among the latter.

Briefly summarizing our findings, we found the computed anharmonicities to be very sensitive to the DFT integration grid and step sizes. We were only able to assess a few different basis sets, and concluded that the TZ2P basis set was likely to be sufficient in all cases. For basis sets of double-zeta quality, the basis set error was on the order of the error inherent in the exchange-correlation functionals themselves, hence results using these basis sets are to be taken with some caution. HCTH/407 appeared to be the most suitable GGA functional for the purpose, and B97-1 the most suitable hybrid GGA functional, immediately followed by B3LYP. Somewhat surprisingly, when combining DFT anharmonicities with large basis set CCSD(T) geometries and harmonic frequencies, GGA functionals yielded better results than their hybrid counterparts. In many cases, ZPVEs (zero-point vibrational energies) and thermal corrections of sufficiently high quality to be used in conjunction with nonempirical extrapolation-based computational thermochemistry methods like W1, W2, and W3 theory [37, 38] — or explicitly correlated methods like CC-R12 [39] — can only be achieved by combining large basis set $\operatorname{CCSD}(\mathrm{T})$ geometries and harmonic frequencies with the DFT anharmonicities.

In the present contribution, we shall assess performance of a wider variety of exchangecorrelation functionals, including several meta-GGA and meta-GGA functionals, as well 
as some recent functionals developed with thermochemical kinetics in mind. We will also include HF and MP2 results for comparison.

In addition, we will consider a much wider variety of basis sets. Suitable step sizes and DFT grids were established in the previous paper and will not be reconsidered here.

\section{COMPUTATIONAL DETAILS}

All calculations were run on the Intel/Linux farms of the Martin group and of the Faculty of Chemistry at the Weizmann Institute of Science.

Following the approach first proposed by Schneider and Thiel 40], a full cubic and a semidiagonal quartic force field are obtained by central numerical differentiation (in rectilinear normal coordinates about the equilibrium geometry) of analytical second derivatives. The latter were obtained by means of locally modified versions of GAUSSIAN 03 41]; modified routines from CADPAC 42] were used as the driver for the numerical differentiation routine. In this approach, the potential energy surface is expanded through quartic terms at the global minimum geometry like:

$$
V=\frac{1}{2} \sum_{i} \omega_{i} q_{i}^{2}+\frac{1}{6} \sum_{i j k} \phi_{i j k} q_{i} q_{j} q_{k}+\frac{1}{24} \sum_{i j k} \phi_{i j k k} q_{i} q_{j} q_{k} q_{l}
$$

where the $q_{i}$ are dimensionless rectangular normal coordinates, $\omega_{i}$ are harmonic frequencies, and $\phi_{i j k}$ and $\phi_{i j k l}$ third and fourth derivatives with respect to the $q_{i}$ at the equilibrium geometry.

All the force fields have been analyzed by means of the SPECTRO[43] and by POLYAD [44] rovibrational perturbation theory programs developed by the Handy group and by Martin, respectively.

In all cases, when strong Fermi resonances lead to band origins perturbed more than about $2 \mathrm{~cm}^{-1}$ from their second-order position, the deperturbed values are reported and resonance matrices diagonalized to obtain the true band origins. Rotational constants were similarly deperturbed for strong Coriolis resonances.

Thermodynamic functions beyond the harmonic approximation are obtained by means of the integration of asymptotic series method as implemented in the NASA PAC99 program of McBride and Gordon[45].

The quadrature grids used are Euler-Maclaurin radial grids 46] with 140 points if the 
molecule includes only first-row atoms, and 200 points otherwise. As angular grid the 974point Lebedev grid[47] was employed. For consistency with our previous paper 20], grid pruning was not applied, although spot-checking suggests that it would not have affected results in any significant way. Such grids are commonly denoted $140 \times 974$ and $200 \times 974$, respectively. For the CPKS (coupled-perturbed Kohn-Sham) steps, we used a different, significantly coarser $(75 \times 194)$ grid. In our previous paper we showed that this combination of grids results in computed fundamental frequencies that are numerically precise to $1 \mathrm{~cm}^{-1}$ or better.

The numerical step size determined to be optimal in our previous work [20] was:

$$
q_{\text {step }}(i)=4 \times \sqrt{\frac{\mu}{\mathrm{amu}}} \times \sqrt{\frac{1000 \mathrm{~cm}^{-1}}{\omega(i)}}
$$

The steps are done along the unnormalized Cartesian displacement vector of the mass weighted normal coordinates.

We have furthermore tightened convergence criteria at all stages of the electronic structure calculations to $10^{-10}$ or better (no convergence could be achieved with even tighter criteria). No auxiliary basis sets were employed.

\section{RESULTS AND DISCUSSION}

\section{A. Basis set}

We have considered the following basis sets (see Table \ for detailed primitive and contracted basis set sizes):

- Dunning's cc-pVnZ correlation consistent basis sets (going from double-zeta to quadruple-zeta quality) for the first row [48], and the cc-pV $(n+\mathrm{d}) \mathrm{Z}$ basis sets of Wilson,

Peterson, and Dunning[49] for the second row. (The latter include additional highexponent $d$ functions, which have been shown to be important 29] for spectroscopic constants of molecules in which a second-row atom is surrounded by one of more highly electronegative first-row atoms.) These basis sets were optimized at the CISD (configuration interaction with all single and double excitations) level.

- The TZ2P [50] and DZP [51] basis sets. (Note that the TZ2P version used by most groups actually includes a third $d$ function for second-row atoms.) 
- Jensen's pc- $n$ basis sets which have been specifically optimized for the BLYP functional [52, 53, 54, 55]. The pc-1 and pc-2 basis sets are the same size as Dunning's cc-pVDZ and cc-pVTZ basis sets, respectively, while the pc-3 basis set is of $5 s 4 p 2 d 1 f$ quality on $\mathrm{H}, 6 s 5 p 3 d 2 f 1 g$ quality on $\mathrm{C}-\mathrm{F}$, and $6 s 5 p 3 d 2 f 1 g$ quality on $\mathrm{Si}-\mathrm{Cl}$, and could arguably be described as a quintuple-zeta rather than a quadruple-zeta basis set.

- Pople's 3-21G [56, 57], 6-31G [58, 59], and 6-311G [60, 61, 62] basis sets (the latter two with various combinations of diffuse and polarisation functions). The $3-21 \mathrm{G}$ and $6-31 \mathrm{G}$ basis sets (and their polarized variants) were optimized at the Hartree-Fock level, the 6-311G basis set at the MP2 level.

- Ahlrichs' TZV basis set [63], which is usually augmented with the polarization functions of Dunning's cc-pVTZ basis set. We denote this combination TZVPP. For second-row systems, it is again strongly recommended to include an additional $d$ function: a basis set satisfying that requirement, and which shall be denoted $\mathrm{TZV}(\mathrm{P}+1) \mathrm{P}$, was obtained by combining Ahlrichs' TZV with the polarization functions from the cc-pV $(\mathrm{T}+\mathrm{d}) \mathrm{Z}$ basis set [49].

Relative CPU times for the hybrid functionals can be approximately gauged from the basis set sizes in Table \. In practice, CPU time scaling is a lot less steep than the theoretical $N^{4}$ thanks to integral screening and various cutoffs, and scaling becomes more favorable as the molecule grows.

Since we are only comparing basis sets, we used two additional molecules which have not been included into the validation set before, $\mathrm{HOO}$ and $\mathrm{CH}_{3}$. Both force fields have previously been computed [64, 65] with great accuracy by ab initio methods. The lowest bending mode $\left(\Pi_{g}\right)$ of acetylene requires the addition of diffuse functions in order to obtain a qualitatively correct anharmonicity — both ab initio 16] and DFT 20] — and hence has been eliminated from consideration here. Overall, this leaves us with 96 harmonic/fundamental frequencies in our validation set.

The most saturated basis set used is, as our experience shows [66], the pc-3 basis set (see above). As the Dunning correlation consistent basis sets were developed for correlated ab initio methods, exponents for the polarization functions (which in their case are more appropriately named angular correlation functions) are biased to the high-exponent region. As a result, they do not appear to be the most efficient choice of basis sets for Hartree-Fock 
or density functional theory, at least for atomization energies [67]. We compare all basis sets to the largest one used. For B3LYP, where we only looked at five basis sets, the most accurate basis set used is the pc-2 basis set, as the results from Table $\llbracket$ show.

Like for atomization energies, the $3-21 \mathrm{G}$ basis set is yielding extremely large errors. Its RMS error compared to our largest basis set is $150 \mathrm{~cm}^{-1}$, and even the anharmonic correction has an RMS error of $30 \mathrm{~cm}^{-1}$. Even worse, some of the force fields yield positive anharmonic corrections. The unpolarized 6-31G basis set fares little better for harmonic and fundamental frequencies. However, unlike the $3-21 \mathrm{G}$ basis set, the anharmonic corrections for most molecules are at least somewhat reasonable. Nevertheless, even here we experienced (albeit small) positive anharmonic corrections for two molecules. The first basis set which gives good results is the $6-31 G^{*}$ basis set. The RMS error for the harmonic and fundamental frequency is still considerable at $46 \mathrm{~cm}^{-1}$, however no more qualitatively wrong anharmonicities are seen. The rest of the double-zeta basis sets seem to give results which are comparable to each other(RMS errors around $30 \mathrm{~cm}^{-1}$ ), whereas the $6-31+\mathrm{G}^{*}$ and pc-1 basis sets are a bit closer to the basis set limit. Starting with basis sets of triple-zeta quality, the basis set errors in the harmonic frequencies get significantly smaller, ranging from 16 to $5 \mathrm{~cm}^{-1}$. Pople's basis sets of triple-zeta quality do not perform well compared to, e.g., the TZ2P basis set, only the $6-311+\mathrm{G}(3 \mathrm{df}, 2 \mathrm{pd})$ with all possible polarization functions shows a low error. The $6-31 \mathrm{G}$ basis sets also seem to require diffuse functions to yield results comparable to the other basis sets double-zeta quality. This reiterates the observation made in our contribution where we looked at basis set effects for atomization energies [67]. Here, we concluded that "diffuse functions are more important with the Pople basis sets, in that they provide a significant error reduction". This implies that the 6-31G basis sets are slightly less balanced than the other used basis sets. When looking at the basis set error of the TZVPP basis set, the extra $d$ polarization function lowers the error by another 30\%, making it the basis set with the lowest error of all triple-zeta basis sets tested. Finally, this error is again reduced by half by the only other basis set of quadruple-zeta quality tested, indicating that the basis set is very close to the Kohn-Sham limit for these properties. Exactly the same behavior can be observed for the B3LYP functional, with similar errors compared to the pc-2 reference basis set. Thus, we can expect all hybrid functionals with $20 \%$ exchange to give similar basis set errors. Interestingly, although the intrinsic basis set errors of different basis sets look like they are the same at the triple-zeta 
level, compared to the pc-2 basis set the error of e.g. $6-311+\mathrm{G}(3 \mathrm{df}, 2 \mathrm{pd})$ basis set is still 8.3 $\mathrm{cm}^{-1}$ ). This implies that the errors for the different triple-zeta basis sets do not seem to be consistent. Relative to pc-3, the average basis set incompleteness error for DFT harmonic or fundamental frequencies obeys the following sequence:

cc-pVQZ $\ll 6-311+\mathrm{G}(3 \mathrm{df}, 2 \mathrm{pd}) \approx \mathrm{TZV}(\mathrm{P}+1) \mathrm{P} \approx \mathrm{pc}-2 \approx \mathrm{TZVPP} \approx \mathrm{cc}-\mathrm{pVTZ}<\mathrm{TZ} 2 \mathrm{P}$ $<6-311+\mathrm{G}(3 \mathrm{~d}, 2 \mathrm{p})<6-311+\mathrm{G}^{* *} \ll \mathrm{pc}-1 \approx 6-31+\mathrm{G}^{* *}<\mathrm{DZP} \approx \mathrm{cc}-\mathrm{pVDZ} \ll 6-31 \mathrm{G}^{*} \ll$ $6-31 G \ll 3-21 G$

Of course, the error in the fundamental frequencies transfers to basis set errors in the zeropoint energies, as shown in Figure 1. Here, considerable error cancellation occurs, and the larger frequencies obtain also a larger weight. For example, the TZ2P basis set has about the same basis set error as 6-311+ $\mathrm{G}^{* *}$, different from what could be deduced from Table [I]. The basis sets of double-zeta quality with polarization functions (excluding 6-31G*) exhibit errors between 0.58 and $0.48 \mathrm{~kJ} / \mathrm{mol}$ compared to the largest basis set used, and the basis sets of triple-zeta quality between 0.38 and $0.26 \mathrm{~kJ} / \mathrm{mol}$.

In Table III, we compare the results of B97-1 and B3LYP with the different basis sets to our reference values. Of course, error cancellation takes place here, and the basis set error of the large basis sets will be masked by the greater error of the functional itself. Nevertheless, from this perspective, the results are a bit surprising: For harmonic frequencies, the TZ2P basis set (which B97-1 has been fitted to) and the DZP basis set(!) show the lowest error in our validation study. For B3LYP, the TZ2P basis set yields the lowest errors as well. Overall, there is little difference between the different basis sets. Only the 3-21G, 6-31G, cc-pVDZ and perhaps the $6-31 \mathrm{G}^{*}$ basis sets exhibit really large errors. For all the other basis sets, basis set error is comparable to the intrinsic error for the exchange-correlation functional. Still, it might be possible that some molecules outside our validation set have a stronger basis set dependence, making at least a basis set of triple-zeta quality necessary. For example, the aforementioned $\mathrm{C}_{2} \mathrm{H}_{2}$ molecule has a strong basis set dependence and showed large errors and a positive anharmonic correction when not using diffuse functions in the basis set. Consistently for all basis sets, B3LYP has a larger RMS error than B97-1 in the harmonic frequencies by about $2 \mathrm{~cm}^{-1}$. On the other hand, the anharmonic correction seems to be described better by the B3LYP functional for most basis sets (with exception 
of the pc-1 basis set, which yields particularly low errors for the anharmonic correction in conjunction with the B97-1 basis set). Nevertheless, the differences are very small, and might well change when larger and other molecules would be considered in the validation set.

We again consider the anharmonic zero-point energies, error statistics for which are shown in Figure 2. Like for the fundamental frequencies, no difference between the basis sets of double and triple-zeta quality can be seen. The TZ2P basis set once more exhibits the most pronounced error cancellation between the basis set error and intrinsic error of the functional.

The basis set errors for the rotational constants (relative to the largest basis set considered, pc-3) are shown in Table IV For the TZ2P basis set, the $\mathrm{CH}_{2}$ molecule has been excluded. The $B_{e}$ values correlate directly to the accuracy of the geometry of the molecule. The difference between the errors of the different $B_{e}$ and $B_{0}$ values is quite small, since only the third derivatives contribute to it. As one might expect, all basis sets underestimate the rotational constants (or, conversely, overestimate bond lengths) relative to the basis set limit. As for the harmonic frequencies, a very large difference is encountered when going from the 3-21G and 6-31G basis sets to those including polarization functions. While the error is cut down to a third by including diffuse functions for harmonic frequencies, for rotational constants it is reduced even further, to approximately one-quarter. Going from double to triple zeta, the same trend can be observed, with errors in both frequencies and rotational constants being cut to approximately one-third. Even without the diffuse functions, the 6-31G* basis set yields a lower error than the pc-1 basis set. Comparing the basis sets of triple-zeta quality, the same trend can be observed. TZ2P seems to be a good compromise between quality and computational cost, as it yields an RMS error lower than the pc-2 basis set despite not containing any $f$ functions. For rotational constants and thus geometries, the extra tight $d$ functions for the TZVPP basis set seems to be extremely important: The RMS error is reduced from $1 \%$ to $0.29 \%$ just by including this additional function for the second-row molecules. While the TZVPP basis set is one of the worstperformers of the triple-zeta basis sets tested, the $\mathrm{TZV}(\mathrm{P}+1) \mathrm{P}$ basis set is only surpassed in accuracy by the $6-311+\mathrm{G}(3 \mathrm{df}, 2 \mathrm{pd})$ basis set. The anharmonic corrections exhibit similar basis set convergence behavior, with the notable exception of the TZVPP basis set, where quite large deviations are seen. Again, the basis set dependencies observed for the B97-1 
functional can be transferred to the RMS errors of the B3LYP functional.

The overall errors compared to our reference values in Table $\nabla$ all consistently underestimate the rotational constants when using either the B3LYP or B97-1 functionals. This implies that, as we have seen above, when getting closer to the basis set limit our structures (at least for our validation set) also become better. As a rule of thumb, this is generally the case for these two most popular hybrid functionals. Hence, unlike the case of frequencies - where double-zeta basis sets sometimes yield closer agreement with experiment than their quadruple-zeta counterparts because of error compensation with the intrinsic error of the functional - larger basis sets always yield better agreement with the reference data for geometries, and at least a polarized triple-zeta basis set is always recommended. This implies that for our test set, an (by a small amount) larger admixture of exchange in the hybrid functional would yield better results. In the next section, such functionals will be assessed. Again, the effect when going from $r_{e}$ to $r_{0}$ geometries is quite small, at least compared to the error made by DFT. All basis sets, even including the 3-21G basis set, yield the same error in the corrections.

For the sake of brevity, we will only report errors in the thermodynamic functions using the different basis sets. All data (Table VI) are relative to thermodynamic functions obtained from our reference spectroscopic constants by the same integrated asymptotic series procedure. Also, we will not discuss here any of the approximations considered in our previous paper, such as the RRHO approximation with equilibrium geometries and harmonic frequencies, the RRHO approximation with zero-point average geometries and fundamental frequencies or using DFT anharmonic force fields combined with large basis set $\operatorname{CCSD}(\mathrm{T})$ geometries and harmonic frequencies. Hence, only the data obtained from the pure DFT anharmonic force field will be presented. Generally, the results of the thermodynamic functions can be understood form the errors of the different basis sets in Table III and Table V] unless error compensation takes place. Unfortunately, this seems to be the case, and it is very hard to draw conclusions. The errors of basis sets of double- and triple-zeta quality show virtually no difference. Only for the entropy, larger deviations between the different basis sets can be seen. In our previous study, it proved to be the only of the three variables under investigation which showed a lower error for the combination of $\operatorname{CCSD}(\mathrm{T})$ harmonic frequencies and geometries with DFT compared to the pure DFT results at $2000 \mathrm{~K}$. This implies that the error cancellation effects do not seem to work as well for the entropy 
than for the enthalpy or the heat capacity.

\section{B. Exchange-correlation functionals}

Turning towards the assessment of density functionals, we used for the functionals the TZ2P basis set for all molecules except $\mathrm{C}_{2} \mathrm{H}_{2}$. For the latter molecule, diffuse functions were necessary, hence the aug-cc-pVTZ basis set was employed in this case. In our previous study, we tested the BLYP, HCTH/407, PBE, B97-1, B97-2, and PBE0 functionals. However, in the last two years some additional functionals have been published, which may replace the old functionals. In addition, we have computed $a b$ initio force fields with the MP2 and HF methods to compare to the functionals tested.

In our experience, CPU times with Gaussian 03 do not strongly, or very systematically, depend on the exchange-correlation functional, especially as no auxiliary "density fitting" basis sets are used here. With other codes, GGAs will offer a marked advantage over hybrid functionals.

The main progress made in recent years in functional development was the inclusion of the kinetic energy density in a couple of functionals. This has been done first by Becke [68], then by Van Voorhis and Scuseria [69] as well as by Boese and Handy [0]. Lately, Perdew and coworkers 71] have also published a new meta-Generalized Gradient Approximation (meta-GGA) functional. The resulting functionals were Bc95 (which however was later disavowed by Becke himself:[72] "The functional of Part IV [i.e., Ref.[68]] is problematic in very weakly bound systems... and is therefore not recommended. This will be corrected in future publications."), VSXC, $\tau$-HCTH and its hybrid and TPSS and its hybrid version.

Several groups realized [3, 74] that typical hybrid GGA functionals (with about 20\% exact exchange) have significant problems in describing barrier heights (albeit less so than pure GGAs). Durant 74] serendipitously found that BHLYP (with 50\% exact exchange 75]) exhibits these problems to a much lesser degree than typical hybrid GGAs. Truhlar and coworkers [6] took an existing modified Perdew-Wang functional [7], 78] and optimized the fraction of Hartree-Fock exchange for optimal reproduction of a representative set of reaction barrier heights. The resulting mPW1K functional had $42.8 \%$ of Hartree-Fock type exchange. However, this functional, like other hybrid GGA functionals with such a large fraction of 'exact' exchange, performs particularly poorly for atomization energies and ground- 
state geometries[67]. Truhlar and coworkers later proposed 82] two functionals around the Bc95 correlation functional, mPW1B95 (with $44 \%$ exact exchange) for equilibrium thermochemistry and mPWB1K (with $31 \%$ exact exchange) for kinetics.

Finally, Boese and Handy conjectured that $\tau$ does not merely simulate exact exchange (as previously argued [69]), but that it is capable of simulating variable exact exchange 70], and thus is potentially capable of substantially reduced errors in equilibrium properties with high-exact exchange functionals. Figure 1 of Ref. 79$]$ proves the latter to be the case, and offers at least circumstantial evidence that the $\tau$ terms can 'back-correct' for excessive exact exchange where it is desirable (that is, near equilibrium geometries). Boese and Martin [9] were thus able to construct a density functional, termed BMK (Boese-Martin for Kinetics) that accurately reproduces transition states and reaction barrier heights without compromising other properties.

Hence, the new functionals tested are the GGA functionals BLYP, HCTH/407, PBE (from our previous paper), the TPSS meta-GGA functional, the hybrid functionals (developed for use of ground-state properties) B3LYP (20\% exact exchange), B97-1 (21 \%), B97-2 (21 \%), PBE0 (25\%), TPSSh (10\%), and mPW1B95 (31\%) and the hybrid functionals (developed for use of transition-state properties) mPW1K (42.8\%), mPWB1K (44\%) and BMK (42 $\%)$.

The use of exact local exchange for hybrid functionals improves the functionals for many properties, [80] as this method selects the correct multiplicative potential of the functional. However, we did not consider using hybrid functionals with local exact exchange in this study simply because such an exchange-correlation potential does not change the energy nor its nuclear derivatives. Hence, all functionals used will yield quite close results when using Hartree-Fock or local exchange. 81]

When analyzing the data in Table VII HCTH/407 shows the lowest RMS errors of all GGA and meta-GGA functionals, while for example BLYP yields errors almost twice as large. Although TPSS is clearly an improvement over PBE, it is still less accurate than $\mathrm{HCTH} / 407$ for our validation set. Interestingly, the anharmonic correction is a lot worse when including the kinetic energy density here, with TPSS yielding an error almost twice as large as the $\mathrm{HCTH} / 407$ or BLYP functionals.

Turning towards the hybrid functionals, TPSSh is barely an improvement over PBE0, and B97-1 is the functional with the lowest errors for harmonic and fundamental frequencies. 
B97-2 shows the lowest RMS error of hybrid functionals for the anharmonic corrections: this may be related to the fact that exchange-correlation potential points were used in its parametrization. This means that the functional development steps include some information about the PES, since the fit to the exchange-correlation-potential means that the functional derivative is evaluated at every gridpoint of the molecule over full space. mPW1B95, while having errors for atomization energies comparable to B3LYP [82, 83], has a much larger error, which can be almost compared to the error of $\mathrm{HCTH} / 407$ functional for the fundamental frequency. BMK yields, as it was reported for a much larger set of harmonic frequencies [79], an RMS error for both harmonic and fundamental frequency comparable to HCTH/407 and mPW1B95. However, it shows a large error for the anharmonic correction, which is only surpassed by the Hartree-Fock method. This of course suggests that the potential energy surface has been changed, as we could have expected from our previous observations. (For instance, in the case of the $\mathrm{H}_{2} \mathrm{~S}$ molecule, BMK underestimates both harmonic stretching frequencies, unlike all other high-exact exchange functionals. However, the anharmonic corrections are underestimated for this molecules, resulting in accidental good agreement with experiment for the fundamentals.) In general, the anharmonic correction is underestimated on average, and the harmonic frequency overestimated. This corresponds to a steepening of the potential energy surface near the minimum, which is effectively what the kinetic energy density terms 'did' to get the atomization energy correct. Thus, these results give further insight into the functional.

We also note that the performance of the different density functionals for anharmonic frequencies is consistent with what we found for much larger sets of harmonic frequencies. 79] Finally, Hartree-Fock unsurprisingly yields extremely large errors for the frequencies, more than three times as large as the worst functional tested and ten times as large as the most accurate functional. The anharmonic correction is not described well either, although the error here is not as large, only three times as bad as the best functional. MP2 shows errors close to $\mathrm{HCTH} / 407$ or BMK for harmonic and fundamental frequencies, but the corrections are still not as accurate as with most density functionals. To investigate the arguably stronger basis set dependence of the MP2, method, we additionally used a larger cc-pVTZ basis set for MP2. The error is reduced by about ten wavenumbers, which is still worse than all hybrid functionals with a lower HF percentage than 40\%. Overall, we would rank the errors of the methods under investigation for harmonic or fundamental frequencies as follows: 
$\mathrm{B} 97-1 \approx \mathrm{B} 97-2 \approx \mathrm{B} 3 \mathrm{LYP} \approx \mathrm{PBE} 0 \approx \mathrm{TPSSh}<\mathrm{mPW} 1 \mathrm{~B} 95<\mathrm{HCTH} / 407 \approx \mathrm{MP} 2 \approx \mathrm{BMK}$ $\approx \mathrm{TPSS} \ll \mathrm{mPWB} 1 \mathrm{~K} \approx \mathrm{mPW} 1 \mathrm{~K} \approx \mathrm{PBE}<\mathrm{BLYP} \ll \mathrm{HF}$

Generally, the errors of the fundamental frequencies transfer to RMS errors of the zeropoint energies of the methods in displayed in Figure 3. Again, B97-1 shows the lowest error of all methods tested, with the error of $\mathrm{HCTH} / 407$, TPSS and BMK about twice as large. Here, MP2 has an RMS error slightly worse than the latter three functionals. For the computation of accurate transition states accurate zero-point energies are needed. Usually, a frequency calculation has to be performed anyhow (in order to verify that one indeed has found a transition state), and good performance of the functional for zero-point energies allows one to 'recycle' that frequency calculation for the ZPVE. Here, we again see a superior performance of BMK over the other two functionals developed for such interactions, reducing the error by about half. This means that the not-so-good performance of $\mathrm{mPW} 1 \mathrm{~K}$ and $\mathrm{mPWB} 1 \mathrm{~K}$ is even worse than expected, since its errors in the zero-point energies are even close to the worst-performer GGA functionals, BLYP and PBE.

The errors of the different functionals for rotational constants, using the TZ2P basis set (again excluding $\mathrm{CH}_{2}$ from the validation set) are displayed in Table VIIII For geometries, the functionals with $\geq 40 \%$ exchange - with the notable exception of BMK - all do worse than the GGA functionals tested. Both TPSS and TPSSh yield much poorer results as well. Otherwise, the results are quite similar to what we observed for the frequencies. HCTH/407 yields the lowest errors of the GGA functionals, and all hybrid functionals with about $20 \%$ exact exchange yield similar results. By just ranking our hybrid functionals by the amount of exact exchange, we can determine their accuracy: TPSSh, with only $10 \%$ exact exchange yields errors worse than the GGA functional HCTH/407. B3LYP and B97-1 have 20 and $2 \%$ exact exchange, respectively. Although B97-2 also has $21 \%$ exact exchange, it shows lower errors than the latter two because it has been fit to exchange-correlation potential points. Finally, PBE0 has 25\% exact exchange, yielding errors for rotational constants close to B97-2. Nevertheless, TPSS is again an improvement over PBE, although the improvement is not as large as it was for harmonic frequencies. For the sake of comparison, MP2 yields results worse than the 'regular exchange' hybrid GGA functionals but better than most GGA functionals, and also better than the high-exact exchange functionals 
(except for BMK). When increasing the basis set for MP2, the errors are approximately lowered to those of the hybrid functionals, although it does not surpass them. Particularly disappointing is performance for both mPW1K and mPW1B95. BMK stands out among the high-exact exchange functionals, yielding RMS errors about half of mPW1K and mPWB1K. Again, the $B_{e}-B_{0}$ corrections are too small to distinguish any functional from each other. All their errors have about the same values, as it was the case when comparing the different basis sets in conjunction with the B97-1 functional to our reference values. Ranking the functionals for the rotational constants, we arrive at:

$\mathrm{PBE} 0 \approx \mathrm{B} 97-2<\mathrm{B} 97-1 \approx \mathrm{B} 3 \mathrm{LYP}<\mathrm{HCTH} / 407 \approx \mathrm{BMK} \approx \mathrm{mPW} 1 \mathrm{~B} 95 \approx \mathrm{MP} 2 \ll \mathrm{TPSSh}$ $<\mathrm{TPSS} \ll \mathrm{mPW} 1 \mathrm{~K} \approx \mathrm{mPWB} 1 \mathrm{~K}<\mathrm{PBE} \ll \mathrm{BLYP} \ll \mathrm{HF}$

The values of three different thermodynamic functions investigated with different functionals are shown in Table IX At room temperature, BMK and MP2 yield the lowest errors of all methods tested. This is changed at $2000 \mathrm{~K}$; as the higher vibrational levels become occupied, the problems of both MP2 and especially BMK become apparent. Since the errors in the anharmonic corrections for both methods are larger than for 'normal' hybrid functionals, they become more inaccurate at higher temperatures. Unlike the results from Table VI] where a lot of error cancellation occurred, most functionals do perform with errors like the aforementioned rankings, at least at the medium temperature of $600 \mathrm{~K}$. Again, the entropy is the most sensitive thermodynamic function to the different methods used.

\section{CONCLUSIONS}

We have assessed a wide variety of basis sets and exchange-correlation functionals for harmonic and fundamental frequencies, rotational constants, and post-RRHO thermodynamic functions.

For harmonic or fundamental vibrational frequencies, even a double-zeta plus polarization basis set will only have a basis set incompleteness error comparable to the intrinsic error in hybrid GGA functionals like B3LYP or B97-1. To be on the safe side, the TZ2P basis set (which in fact is a TZ3P basis set for second-row atoms) can be used. With double-zeta plus polarization basis sets, some error cancellation between basis set and functional errors 
often occurs.

No such cancellation is seen for geometries or rotational constants: Improving the basis set further is always 'worthwhile' here. The TZ2P basis set represents a good compromise between accuracy and computational cost.

No clear conclusions could be drawn for thermodynamic functions, except perhaps for some basis set dependence for entropies at high temperatures.

When using the Ahlrichs TZVPP basis set, performance for second-row molecules can be greatly improved by adding a high-exponent $d$ function, leading to the TZV $(\mathrm{P}+1) \mathrm{P}$ basis set. In general, a high-exponent $d$ function on second-row atoms is strongly recommended.

For harmonic or fundamental frequencies, there is little to choose between the various 'conventional' hybrid GGAs, all of which have intrinsic RMS errors in the $30-35 \mathrm{~cm}^{-1}$ range. (We note that our dataset is somewhat biased towards 'inorganic' molecules, and better DFT performance can be expected for organic molecules.) Somewhat larger RMS errors in the 50 $\mathrm{cm}^{-1}$ range are seen for MP2, HCTH407, TPSS, and BMK. 'Kinetics' functionals (except for BMK), as well as first-generation GGAs, yield much poorer results. One downside of BMK are larger errors than GGAs and 'conventional' hybrid GGAs for anharmonic corrections. MP2 exhibits the same problem to a lesser extent. Analogous conclusions can be drawn for rotational constants, geometries, and thermodynamic functions.

\section{ACKNOWLEDGMENTS}

ADB acknowledges a postdoctoral fellowship from the Feinberg Graduate School (Weizmann Institute). Research at Weizmann was supported by the Minerva Foundation, Munich, Germany, by the Lise Meitner-Minerva Center for Computational Quantum Chemistry (of which JMLM is a member ad personam), and by the Helen and Martin Kimmel Center for Molecular Design. This work is related to Project 2003-024-1-100, "Selected Free Radicals and Critical Intermediates: Thermodynamic Properties from Theory and Experiment," of the International Union of Pure and Applied Chemistry (IUPAC). 
[1] J. M. L. Martin, J. P. François, and R. Gijbels, J. Chem. Phys. 96, 7633 (1992); J. M. L. Martin, unpublished CCSD(T)/cc-pVQZ calculations

[2] S. Dressler and W. Thiel. Chem. Phys. Lett. 273, 71 (1997).

[3] M. Rosenstock, P. Rosmus, E. A. Reinsch, O. Treutler, S. Carter, and N. C. Handy, Mol. Phys. 93, 853 (1998).

[4] A. Miani, E. Cane, P. Palmieri, A. Trombetti, and N. C. Handy, J. Chem. Phys. 112, 248 (2000).

[5] I. Baraille, C. Larrieau, A. Dargelos, and M. Chaillet, Chem. Phys. 271, 91 (2001).

[6] R. Burcl, N. C. Handy, and S. Carter, Spectrochim. Acta A 59, 1881 (2003).

[7] A. Caligiana, V. Aquilanti, R. Burcl, N. C. Handy, and D. P. Tew, Chem. Phys. Lett. 369, 335 (2003).

[8] V. Barone, J. Phys. Chem. A 108, 4146 (2004).

[9] A. D. Boese and J. M. L. Martin, J. Phys. Chem. A 108, 3085 (2004).

[10] J. Neugebauer and B. A. Hess, J. Chem. Phys. 118, 7215 (2003).

[11] V. Barone, J. Chem. Phys. 120, 3059 (2004).

[12] P. Carbonniére and V. Barone, Chem. Phys. Lett. 392, 365 (2004).

[13] P. Carbonniére and V. Barone, Chem. Phys. Lett. 399, 226 (2004).

[14] V. Barone, J. Chem. Phys. 122, 014108 (2005).

[15] P. Carbonnière, T. Lucca, C. Pouchan, N. Rega, and V. Barone, J. Comp. Chem. 26, 384 (2005).

[16] J. M. L. Martin, T. J. Lee, and P. R. Taylor, J. Chem. Phys. 108, 676 (1998).

[17] J. M. L. Martin, T. J. Lee, P. R. Taylor, and J. P. François, J. Chem. Phys. 103, 2589 (1995);

J. M. L. Martin and P. R. Taylor, Chem. Phys. Lett. 248, 336 (1996).

[18] J. Demaison, L. Margulès, J. M. L. Martin, and J. E. Boggs, Phys. Chem. Chem. Phys. 4, 3282 (2002).

[19] G. de Oliveira, J. M. L. Martin, I. K. C. Silwal, and J. F. Liebman, J. Comp. Chem. 22, 1297 (2001).

[20] A. D. Boese, W. Klopper, and J. M. L. Martin, Mol. Phys. 103, XXXX (2005). 
[21] T. J. Lee, J. M. L. Martin, and P. R. Taylor, J. Chem. Phys. 102, 254 (1995).

[22] J. M. L. Martin, T. J. Lee, and P. R. Taylor, J. Mol. Spect. 160, 105 (1993); J. M. L. Martin, $\operatorname{CCSD}(\mathrm{T}) / \mathrm{PVQZ}$ calculations.

[23] J. M. L. Martin, J. P. François, and R. Gijbels, J. Mol. Spectr. 169, 445 (1995).

[24] J. M. L. Martin and P. R. Taylor, Chem. Phys. Lett. 205, 535 (1993).

[25] J. M. L. Martin, J. P. François, and R. Gijbels, J. Chem. Phys. 97, 3530 (1992).

[26] D. Wang, Q. Shi, and Q.-S. Zhu, J. Chem. Phys. 112, 9624 (2000).

[27] X.-G. Wang, E. L. Sibert III, and J. M. L. Martin, J. Chem. Phys. 112, 1353 (2000).

[28] J. M. L. Martin, K. K. Baldridge, and T. J. Lee, Mol. Phys. 97, 945 (1999).

[29] J. M. L. Martin, J. Chem. Phys. 108, 2791 (1998).

[30] A. D. Becke, Phys. Rev. A 383098 (1988); C. Lee, W. Yang, R. G. Parr, Phys. Rev. B 37 785 (1988).

[31] A. D. Boese and N. C. Handy, J. Chem. Phys. 114, 5497 (2001).

[32] J. P. Perdew, K. Burke, and M. Ernzerhof, Phys. Rev. Lett. 77, 3865 (1996).

[33] A. D. Becke, J. Chem. Phys. 98, 5648 (1993).

[34] F. A. Hamprecht, A. J. Cohen, D. J. Tozer and N. C. Handy, J. Chem. Phys. 109, 6264 (1998).

[35] P. J. Wilson, T. J. Bradley, and D. J. Tozer, J. Chem. Phys. 115, 9233 (2001).

[36] C. Adamo and V. Barone, Chem. Phys. Lett. 298, 113 (1998).

[37] J. M. L. Martin and G. de Oliveira, J. Chem. Phys. 111, 1843 (1999).

[38] A. D. Boese, M. Oren, O. Atasoylu, J. M. L. Martin, M. Kállay and J. Gauss, J. Chem. Phys. 120, 4129 (2004).

[39] For a review see W. Klopper, "R12 methods, Gaussian geminals", in Modern Methods and Algorithms of Quantum Chemistry, 2nd Edition, Ed. J. Grotendorst (John von Neumann Institute for Computing, Jülich, Germany, 2000, pp. 181-229); available online at http://www.fz-juelich.de/nic-series/Volume3/klopper.pdf.

[40] W. Schneider and W. Thiel, Chem. Phys. Lett. 157, 367 (1989).

[41] Gaussian 03, Revision B.02, M. J. Frisch, G. W. Trucks, H. B. Schlegel, G. E. Scuseria, M. A. Robb, J. R. Cheeseman, J. A. Montgomery, Jr., T. Vreven, K. N. Kudin, J. C. Burant, J. M. Millam, S. S. Iyengar, J. Tomasi, V. Barone, B. Mennucci, M. Cossi, G. Scalmani, N. Rega, G. A. Petersson, H. Nakatsuji, M. Hada, M. Ehara, K. Toyota, R. Fukuda, J. Hasegawa, 
M. Ishida, T. Nakajima, Y. Honda, O. Kitao, H. Nakai, M. Klene, X. Li, J. E. Knox, H. P. Hratchian, J. B. Cross, C. Adamo, J. Jaramillo, R. Gomperts, R. E. Stratmann, O. Yazyev, A. J. Austin, R. Cammi, C. Pomelli, J. W. Ochterski, P. Y. Ayala, K. Morokuma, G. A. Voth, P. Salvador, J. J. Dannenberg, V. G. Zakrzewski, S. Dapprich, A. D. Daniels, M. C. Strain, O. Farkas, D. K. Malick, A. D. Rabuck, K. Raghavachari, J. B. Foresman, J. V. Ortiz, Q. Cui, A. G. Baboul, S. Clifford, J. Cioslowski, B. B. Stefanov, G. Liu, A. Liashenko, P. Piskorz, I. Komaromi, R. L. Martin, D. J. Fox, T. Keith, M. A. Al-Laham, C. Y. Peng, A. Nanayakkara, M. Challacombe, P. M. W. Gill, B. Johnson, W. Chen, M. W. Wong, C. Gonzalez, and J. A. Pople, Gaussian, Inc., Pittsburgh PA, 2003.

[42] The Cambridge Analytic Derivatives Package (Cadpac), Issue 6.5, Cambridge, 1998 Developed by R. D. Amos with contributions from I. L. Alberts, J. S. Andrews, S. M. Colwell, N. C. Handy, D. Jayatikala, P.J. Knowles, R. Kobayashi,K. E. Laidig, G. Laming, A. M. Lee, P. E. Maslen, C. W. Murray, P. Palmieri, J. E. Rice, E. D. Simandiras, A. J. Stone, M.-D. Su, and D. J. Tozer.

[43] J. F. Gaw, A. Willets, W. H. Green, and N. C. Handy, in: J. M. Bowman (Ed.), Advances in Molecular Vibration and Collision Dynamics, JAI Press, Greenwich, CT, 1990.

[44] J. M. L. Martin, "POLYAD: a vibrational perturbation theory program including arbitrary resonance matrices" (Weizmann Institute of Science, Rehovot, 1997).

[45] B. J. McBride and S. Gordon, PAC99, Properties and Coefficients code, NASA Reference Publication RP-1271, NASA Lewis/Glenn Research Center, Cleveland, Ohio, 1992.

[46] C. W. Murray, N. C. Handy, and G. J. Laming, Mol. Phys 78, 997 (1993)

[47] V. I. Lebedev, Zh. Vychisl. Mat. Mat. Fiz. 15, 48 (1975); V. I. Lebedev, Zh. Vychisl. Mat. Mat. Fiz. 16, 293 (1976); V. I. Lebedev, Sibirsk. Mat. Zh. 18, 132 (1977); V. I. Lebedev and A. L. Skorokhodov, Russian Acad. Sci. Dokl. Math. 45, 587 (1992)

[48] T. H. Dunning, J. Chem. Phys. 90, 1007 (1989); R. A. Kendall, T. H. Dunning, and R. J. Harrison, J. Chem. Phys. 96, 6796 (1992).

[49] A. K. Wilson, K. A. Peterson, and T. H. Dunning Jr., J. Chem. Phys. 114, 9244 (2001).

[50] T. H. Dunning, J. Chem. Phys. 55, 716 (1971).

[51] T. H. Dunning Jr. and P. J. Hay, in: Modern Theoretical Chemistry, ed. H. F. Schaefer III, Plenum, New York (1976), vol.3, 1.

[52] F. Jensen, J. Chem. Phys. 115, 9113 (2001); erratum 116, 3502 (2002). 
[53] F. Jensen, J. Chem. Phys. 116, 7372 (2002).

[54] F. Jensen, J. Chem. Phys. 117, 9234 (2002).

[55] F. Jensen and T. Helgaker, J. Chem. Phys. 121, 3463 (2004).

[56] J. S. Binkley, J. A. Pople, and W. J. Hehre, J. Am. Chem. Soc. 102, 939 (1980).

[57] M. S. Gordon, J. S. Binkley, J. A. Pople, W. J. Pietro, and W. J. Hehre, J. Am. Chem. Soc. 104, 5039 (1982).

[58] W. J. Ditchfield and J. A. Pople, J. Chem. Phys. 56, 2257 (1971).

[59] P. C. Hariharan and J. A. Pople, Theor. Chim. Acta 28, 213 (1973).

[60] M. J. Frisch, J. A. Pople, and J. S. Binkley, J. Chem. Phys. 80, 3265 (1984).

[61] R. Krishnan, J. S. Binkley, R. Seeger, and J. A. Pople, Theor. Chem. Acc. 72, 650 (1980).

[62] A. D. McLean and G. S. Chandler, J. Chem. Phys. 72, 5639 (1980).

[63] A. Schäfer, C. Huber, and R. Ahlrichs, J. Chem. Phys. 100, 5829 (1994).

[64] D. W. Schwenke, Spectrochim. Acta A 55, 731 (1999).

[65] B. A. Flowers, P. G. Szalay, J. F. Stanton, M. Kállay, J. Gauss, and A. G. Császár, J. Phys. Chem. A, 108, 3195 (2004).

[66] A. D. Boese, J. M. L. Martin, and N. C. Handy, unpublished results.

[67] A. D. Boese, J. M. L. Martin, and N. C. Handy, J. Chem. Phys. 119, 3005 (2003).

[68] A. D. Becke, J. Chem. Phys. 104, 1040 (1996).

[69] T. Van Voorhis, and G. E. Scuseria, J. Chem. Phys 109, 400 (1998).

[70] A. D. Boese and N.C. Handy, J. Chem. Phys. 116, 9559 (2002).

[71] J. M. Tao, J. P. Perdew, V. N. Staroverov, and G. E. Scuseria, Phys. Rev. Lett. 91, 146401 (2003).

[72] A. D. Becke, J. Chem. Phys. 107, 8554 (1997).

[73] J. Baker, J. Andzelm, M. Muir, and P. R. Taylor, Chem. Phys. Lett. 237, 53 (1995).

[74] J. L. Durant, Chem. Phys. Lett. 256, 595 (1996).

[75] A. D. Becke, J. Chem. Phys. 98, 1394 (1993).

[76] B. J. Lynch, P. L. Fast, M. Harris, and D. G. Truhlar, J. Phys. Chem. A 104, 4811 (2000).

[77] J. P. Perdew and Y. Wang, Phys. Rev. B. 4513244 (1992).

[78] C. Adamo and V. Barone, J. Chem. Phys. 108, 664 (1998).

[79] A. D. Boese and J. M. L. Martin, J. Chem. Phys. 121, 3405 (2004).

[80] W. Hieringer, F. Della Sala, and A. Görling Chem. Phys. Lett. 383, 115 (2004). 
[81] V. V. Karasiev, J. Chem. Phys. 118, 8576 (2003).

[82] Y. Zhao and D. G. Truhlar, J. Phys. Chem. A 108, 6908 (2004).

[83] Y. Zhao, B. J. Lynch, and D. G. Truhlar, Phys. Chem. Chem. Phys. 7, 43 (2005). 


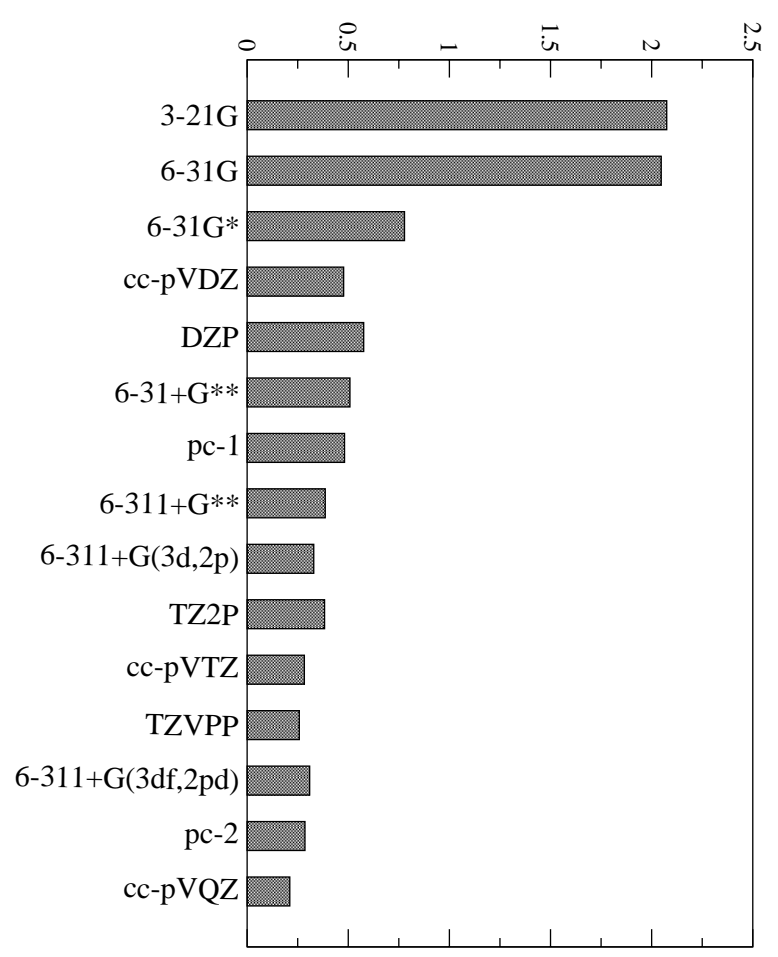

FIG. 1: Boese et al 


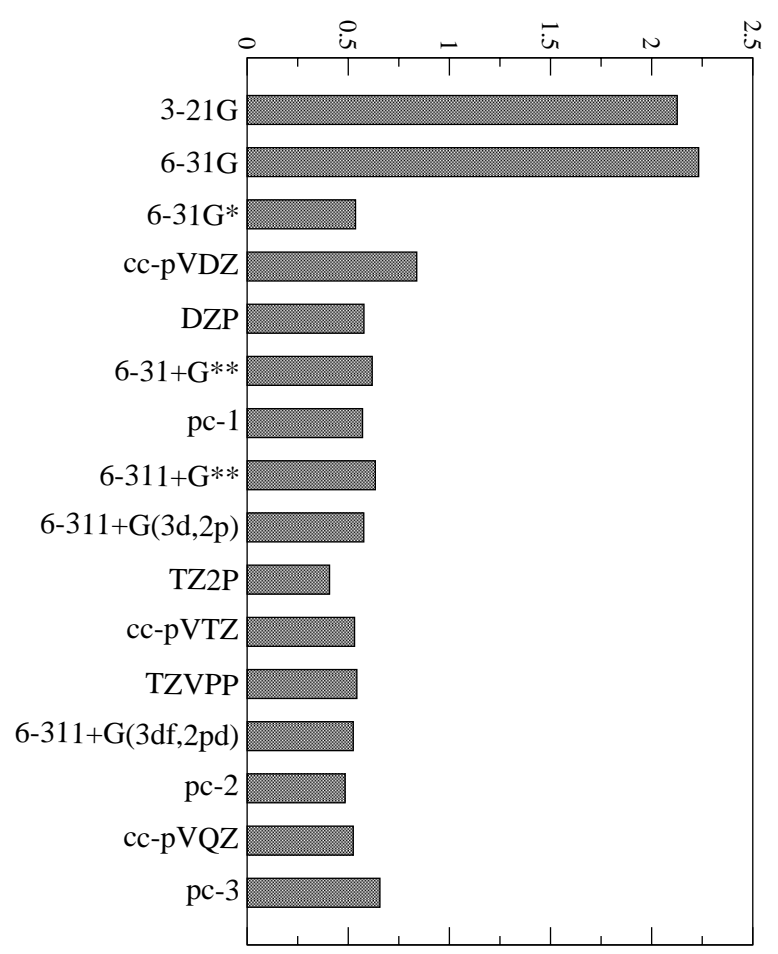

FIG. 2: Boese et al 


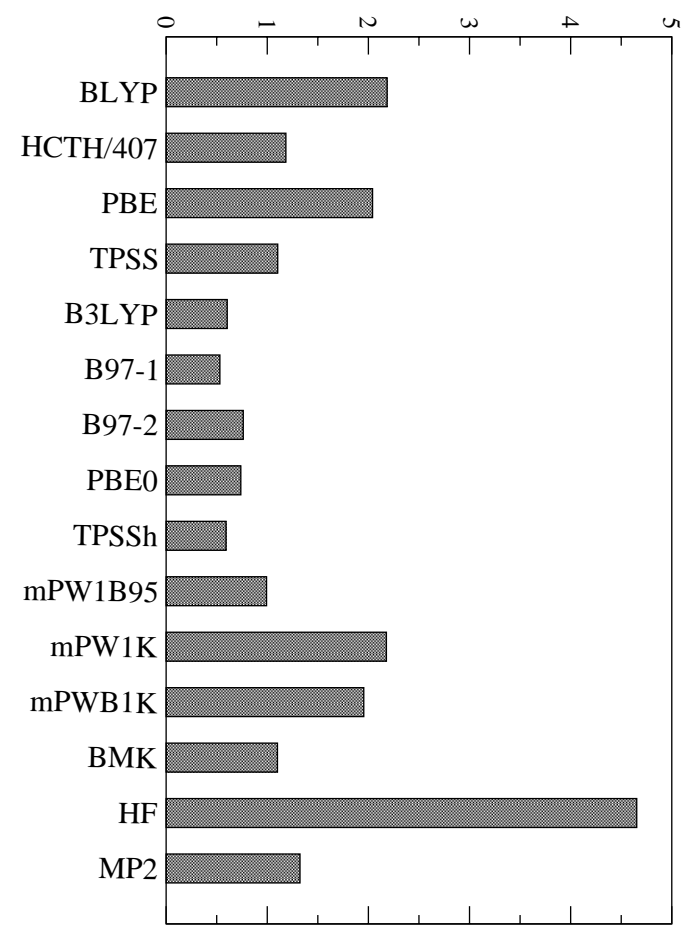

FIG. 3: Boese et al 
Fig. 1:

RMS errors (in $\mathrm{kJ} / \mathrm{mol}$ ) for the B97-1 anharmonic ZPVE for several basis sets, relative to the largest basis set used.

Fig. 2:

RMS errors (in $\mathrm{kJ} / \mathrm{mol}$ ) for the B97-1 anharmonic ZPVE for several basis sets, relative to the $a b$ initio reference values.

Fig. 3:

RMS errors (in $\mathrm{kJ} / \mathrm{mol}$ ) for several functionals for the anharmonic ZPVE, compared to the $a b$ initio reference values. 
TABLE I: Summary of basis sets used in the present work.

\begin{tabular}{|c|c|c|c|c|c|c|c|c|c|}
\hline \multirow[b]{3}{*}{$3-21 G$} & \multicolumn{3}{|c|}{ Uncontracted } & \multicolumn{3}{|c|}{ Contracted } & \multirow{2}{*}{\multicolumn{3}{|c|}{$\begin{array}{l}\text { \# basis functions } \\
\text { H 1st row 2nd row }\end{array}$}} \\
\hline & \multirow{2}{*}{$\frac{\mathrm{H}}{3 \mathrm{~s}}$} & \multirow{2}{*}{$\frac{1 \text { st row }}{6 \mathrm{~s} 3 \mathrm{p}}$} & \multirow{2}{*}{$\frac{2 \text { nd row }}{9 \mathrm{~s} 6 \mathrm{p}}$} & \multirow{2}{*}{$\frac{\mathrm{H}}{2 \mathrm{~s}}$} & \multirow{2}{*}{$\frac{1 \text { st row }}{3 \mathrm{~s} 2 \mathrm{p}}$} & \multirow{2}{*}{$\frac{2 \text { nd row }}{4 \mathrm{~s} 3 \mathrm{p}}$} & & & \\
\hline & & & & & & & 2 & 9 & 13 \\
\hline $6-31 \mathrm{G}$ & $6 \mathrm{~s}$ & $10 \mathrm{~s} 4 \mathrm{p}$ & $16 \mathrm{~s} 10 \mathrm{p}$ & $2 \mathrm{~s}$ & $3 \mathrm{~s} 2 \mathrm{p}$ & $4 \mathrm{~s} 3 \mathrm{p}$ & 2 & 9 & 13 \\
\hline $6-31 \mathrm{G}^{*}$ & $6 \mathrm{~s}$ & $10 \mathrm{~s} 4 \mathrm{p} 1 \mathrm{~d}$ & 16s10p1d & $2 \mathrm{~s}$ & $3 \mathrm{~s} 2 \mathrm{p} 1 \mathrm{~d}$ & $4 \mathrm{~s} 3 \mathrm{p} 1 \mathrm{~d}$ & 2 & $15^{a}$ & $19^{a}$ \\
\hline $6-31+\mathrm{G}^{* *}$ & $6 \mathrm{~s} 1 \mathrm{p}$ & 11s5p1d & $17 \mathrm{~s} 11 \mathrm{p} 1 \mathrm{~d}$ & $2 \mathrm{~s} 1 \mathrm{p}$ & $4 \mathrm{~s} 3 \mathrm{p} 1 \mathrm{~d}$ & $4 \mathrm{~s} 3 \mathrm{p} 1 \mathrm{~d}$ & 5 & $19^{a}$ & $23^{a}$ \\
\hline $6-311+\mathrm{G}^{* *}(\mathrm{~b})$ & $5 \mathrm{~s} 1 \mathrm{p}$ & $12 \mathrm{~s} 6 \mathrm{p} 1 \mathrm{~d}$ & $14 \mathrm{~s} 10 \mathrm{p} 1 \mathrm{~d}$ & $3 \mathrm{~s} 1 \mathrm{p}$ & $5 \mathrm{~s} 4 \mathrm{p} 1 \mathrm{~d}$ & $7 \mathrm{~s} 6 \mathrm{p} 1 \mathrm{~d}$ & 6 & 22 & 30 \\
\hline $6-311+\mathrm{G}(3 \mathrm{~d}, 2 \mathrm{p})$ & $5 \mathrm{~s} 2 \mathrm{p}$ & $12 \mathrm{~s} 6 \mathrm{p} 3 \mathrm{~d}$ & $14 \mathrm{~s} 10 \mathrm{p} 3 \mathrm{~d}$ & $3 \mathrm{~s} 2 \mathrm{p}$ & 5s4p3d & $7 \mathrm{~s} 6 \mathrm{p} 3 \mathrm{~d}$ & 9 & 32 & 40 \\
\hline $6-311+\mathrm{G}(3 \mathrm{df}, 2 \mathrm{pd})$ & $5 \mathrm{~s} 2 \mathrm{p} 1 \mathrm{~d}$ & $12 \mathrm{~s} 6 \mathrm{p} 3 \mathrm{~d} 1 \mathrm{f}$ & 14s10p3d1f & $3 \mathrm{~s} 2 \mathrm{p} 1 \mathrm{~d}$ & $5 \mathrm{~s} 4 \mathrm{p} 3 \mathrm{~d} 1 \mathrm{f}$ & 7s6p3d1f & 14 & 39 & 47 \\
\hline DZP & $4 \mathrm{~s} 1 \mathrm{p}$ & 9s5p1d & 11s7p1d & $2 \mathrm{~s} 1 \mathrm{p}$ & $4 \mathrm{~s} 2 \mathrm{p} 1 \mathrm{~d}$ & $6 \mathrm{~s} 4 \mathrm{p} 1 \mathrm{~d}$ & 5 & 16 & 24 \\
\hline TZ2P & $5 \mathrm{~s} 2 \mathrm{p}$ & $10 \mathrm{~s} 6 \mathrm{p} 2 \mathrm{~d}$ & $12 \mathrm{~s} 9 \mathrm{p} 3 \mathrm{~d}$ & $3 \mathrm{~s} 2 \mathrm{p}$ & $5 \mathrm{~s} 4 \mathrm{p} 2 \mathrm{~d}$ & $9 \mathrm{~s} 6 \mathrm{p} 3 \mathrm{~d}$ & 9 & 27 & 42 \\
\hline cc-pVDZ & $4 \mathrm{~s} 1 \mathrm{p}$ & $9 \mathrm{~s} 4 \mathrm{p} 1 \mathrm{~d}$ & $12 \mathrm{~s} 8 \mathrm{p} 1 \mathrm{~d}$ & $2 \mathrm{~s} 1 \mathrm{p}$ & $3 \mathrm{~s} 2 \mathrm{p} 1 \mathrm{~d}$ & $4 \mathrm{~s} 3 \mathrm{p} 1 \mathrm{~d}$ & 5 & 14 & 18 \\
\hline cc-pVTZ & $5 \mathrm{~s} 2 \mathrm{p} 1 \mathrm{~d}$ & 10s5p2d1f & $15 \mathrm{~s} 9 \mathrm{p} 2 \mathrm{~d} 1 \mathrm{f}$ & $3 \mathrm{~s} 2 \mathrm{p} 1 \mathrm{~d}$ & $4 \mathrm{~s} 3 \mathrm{p} 2 \mathrm{~d} 1 \mathrm{f}$ & $5 \mathrm{~s} 4 \mathrm{p} 2 \mathrm{~d} 1 \mathrm{f}$ & 14 & 30 & 34 \\
\hline cc-pVQZ & $6 \mathrm{~s} 3 \mathrm{p} 2 \mathrm{~d} 1 \mathrm{f}$ & 12s6p3d2f1g & 16s11p3d2f1g & $4 \mathrm{~s} 3 \mathrm{p} 2 \mathrm{~d} 1 \mathrm{f}$ & $5 \mathrm{~s} 4 \mathrm{p} 3 \mathrm{~d} 2 \mathrm{f} 1 \mathrm{~g}$ & $6 \mathrm{~s} 5 \mathrm{p} 3 \mathrm{~d} 2 \mathrm{f} 1 \mathrm{~g}$ & 30 & 55 & 59 \\
\hline$c c-p V(D+d) Z$ & $4 \mathrm{~s} 1 \mathrm{p}$ & $9 \mathrm{~s} 4 \mathrm{p} 1 \mathrm{~d}$ & $12 \mathrm{~s} 8 \mathrm{p} 2 \mathrm{~d}$ & $2 \mathrm{~s} 1 \mathrm{p}$ & $3 \mathrm{~s} 2 \mathrm{p} 1 \mathrm{~d}$ & $4 \mathrm{~s} 3 \mathrm{p} 2 \mathrm{~d}$ & 5 & 14 & 23 \\
\hline cc-pV $(T+d) Z$ & $5 \mathrm{~s} 2 \mathrm{p} 1 \mathrm{~d}$ & $10 \mathrm{~s} 5 \mathrm{p} 2 \mathrm{~d} 1 \mathrm{f}$ & 15s9p3d1f & $3 \mathrm{~s} 2 \mathrm{p} 1 \mathrm{~d}$ & $4 \mathrm{~s} 3 \mathrm{p} 2 \mathrm{~d} 1 \mathrm{f}$ & $5 \mathrm{~s} 4 \mathrm{p} 3 \mathrm{~d} 1 \mathrm{f}$ & 14 & 30 & 39 \\
\hline$c c-p V(Q+d) Z$ & $6 \mathrm{~s} 3 \mathrm{p} 2 \mathrm{~d} 1 \mathrm{f}$ & 12s6p3d2f1g & $16 \mathrm{~s} 11 \mathrm{p} 4 \mathrm{~d} 2 \mathrm{f} 1 \mathrm{~g}$ & $4 \mathrm{~s} 3 \mathrm{p} 2 \mathrm{~d} 1 \mathrm{f}$ & $5 \mathrm{~s} 4 \mathrm{p} 3 \mathrm{~d} 2 \mathrm{f} 1 \mathrm{~g}$ & $6 \mathrm{~s} 5 \mathrm{p} 4 \mathrm{~d} 2 \mathrm{f} 1 \mathrm{~g}$ & 30 & 55 & 64 \\
\hline pc-1 & $4 \mathrm{~s} 1 \mathrm{p}$ & $7 \mathrm{~s} 4 \mathrm{p} 1 \mathrm{~d}$ & 11s8p1d & $2 \mathrm{~s} 1 \mathrm{p}$ & $3 \mathrm{~s} 2 \mathrm{p} 1 \mathrm{~d}$ & $4 \mathrm{~s} 3 \mathrm{p} 1 \mathrm{~d}$ & 5 & 14 & 18 \\
\hline pc-2 & $6 \mathrm{~s} 2 \mathrm{p} 1 \mathrm{~d}$ & $10 \mathrm{~s} 6 \mathrm{p} 2 \mathrm{~d} 1 \mathrm{f}$ & $13 \mathrm{~s} 10 \mathrm{p} 2 \mathrm{~d} 1 \mathrm{f}$ & $3 \mathrm{~s} 2 \mathrm{p} 1 \mathrm{~d}$ & $4 \mathrm{~s} 3 \mathrm{p} 2 \mathrm{~d} 1 \mathrm{f}$ & $5 \mathrm{~s} 4 \mathrm{p} 2 \mathrm{~d} 1 \mathrm{f}$ & 14 & 30 & 34 \\
\hline pc-3 & $9 \mathrm{~s} 4 \mathrm{p} 2 \mathrm{~d} 1 \mathrm{f}$ & 14s9p4d2f1g & 17s13p4d2f1g & $5 \mathrm{~s} 4 \mathrm{p} 2 \mathrm{~d} 1 \mathrm{f}$ & $6 \mathrm{~s} 5 \mathrm{p} 4 \mathrm{~d} 2 \mathrm{f} 1 \mathrm{~g}$ & $6 \mathrm{~s} 5 \mathrm{p} 4 \mathrm{~d} 2 \mathrm{f} 1 \mathrm{~g}$ & 34 & 64 & 64 \\
\hline TZVPP & $5 \mathrm{~s} 2 \mathrm{p} 1 \mathrm{~d}$ & $11 \mathrm{~s} 6 \mathrm{p} 2 \mathrm{~d} 1 \mathrm{f}$ & $14 \mathrm{~s} 9 \mathrm{p} 2 \mathrm{~d} 1 \mathrm{f}$ & $3 \mathrm{~s} 2 \mathrm{p} 1 \mathrm{~d}$ & $5 \mathrm{~s} 3 \mathrm{p} 2 \mathrm{~d} 1 \mathrm{f}$ & $5 \mathrm{~s} 4 \mathrm{p} 2 \mathrm{~d} 1 \mathrm{f}$ & 14 & 31 & 34 \\
\hline $\mathrm{TZV}(\mathrm{P}+1) \mathrm{P}$ & $5 \mathrm{~s} 2 \mathrm{p} 1 \mathrm{~d}$ & 11s6p2d1f & 14s9p3d1f & $3 \mathrm{~s} 2 \mathrm{p} 1 \mathrm{~d}$ & $5 \mathrm{~s} 3 \mathrm{p} 2 \mathrm{~d} 1 \mathrm{f}$ & $5 \mathrm{~s} 4 \mathrm{p} 3 \mathrm{~d} 1 \mathrm{f}$ & 14 & 31 & 39 \\
\hline
\end{tabular}

(a) Using Cartesian d functions by default

(b) 2nd row basis set is actually McLean-Chandler 62 
TABLE II: Basis set incompleteness errors $\left(\mathrm{cm}^{-1}\right.$, relative to the pc-3 basis set for B97-1 and to the pc-2 basis set for B3LYP) in harmonic frequencies, fundamental frequencies, and anharmonic corrections for several basis sets used in conjunction with the B97-1 and B3LYP functionals.

\begin{tabular}{|c|c|c|c|c|c|c|c|}
\hline \multicolumn{2}{|c|}{ Property } & \multicolumn{2}{|c|}{ Harmonic Frequency } & \multicolumn{2}{|c|}{ Fundamental Frequency } & \multicolumn{2}{|c|}{ Correction } \\
\hline & Basis set & mean & RMS & mean & RMS & mean & RMS \\
\hline \multicolumn{8}{|c|}{ B97-1 } \\
\hline minimal & $3-21 G$ & -31.3 & 148.1 & -26.2 & 141.1 & -3.2 & 30.0 \\
\hline \multirow[t]{6}{*}{ double-zeta } & $6-31 \mathrm{G}$ & -31.8 & 121.9 & -30.1 & 116.9 & 0.6 & 11.6 \\
\hline & $6-31 \mathrm{G}^{*}$ & 12.9 & 46.6 & 14.3 & 46.6 & 0.4 & 9.8 \\
\hline & cc-pVDZ & -9.3 & 30.7 & -7.4 & 31.5 & -0.1 & 8.9 \\
\hline & $\mathrm{DZP}$ & 2.0 & 29.8 & 3.5 & 27.3 & 0.3 & 5.2 \\
\hline & $6-31+\mathrm{G}^{* *}$ & 3.1 & 26.5 & 4.2 & 26.3 & 0.7 & 4.5 \\
\hline & pc-1 & -1.0 & 26.3 & -0.1 & 27.3 & 0.8 & 6.8 \\
\hline \multirow[t]{8}{*}{ triple-zeta } & $6-311+\mathrm{G}^{* *}$ & -4.3 & 15.6 & -2.3 & 17.1 & -0.2 & 3.6 \\
\hline & $6-311+\mathrm{G}(3 \mathrm{~d}, 2 \mathrm{p})$ & -1.3 & 11.2 & 1.0 & 14.4 & -0.5 & 3.7 \\
\hline & $\mathrm{TZ2P}$ & 0.6 & 8.9 & 3.0 & 13.0 & -0.6 & 3.4 \\
\hline & cc-pVTZ & -0.5 & 6.8 & 1.9 & 9.7 & -0.6 & 2.9 \\
\hline & TZVPP & -0.2 & 6.8 & 1.2 & 8.9 & 0.4 & 2.7 \\
\hline & pc-2 & 1.3 & 6.3 & 2.8 & 8.6 & 0.3 & 2.2 \\
\hline & $6-311+\mathrm{G}(3 \mathrm{df}, 2 \mathrm{pd})$ & 0.7 & 5.2 & 3.1 & 8.8 & -0.5 & 3.1 \\
\hline & $\mathrm{TZV}(\mathrm{P}+1) \mathrm{P}$ & 1.1 & 4.4 & 2.6 & 6.9 & 0.3 & 2.8 \\
\hline quadruple-zeta & cc-pVQZ & 0.3 & 2.8 & 1.9 & 5.4 & 0.2 & 1.5 \\
\hline \multicolumn{8}{|c|}{ B3LYP } \\
\hline \multirow[t]{2}{*}{ double-zeta } & $6-31 G^{*}$ & 10.1 & 45.2 & 9.6 & 42.2 & 0.5 & 10.0 \\
\hline & $6-31+\mathrm{G}^{* *}$ & -0.1 & 21.1 & -0.6 & 20.1 & 0.7 & 3.8 \\
\hline \multirow[t]{3}{*}{ triple-zeta } & $\mathrm{TZ2P}$ & 0.7 & 9.8 & 1.3 & 9.8 & -0.6 & 2.5 \\
\hline & cc-pVTZ & -1.6 & 9.1 & -1.7 & 10.0 & 0.1 & 4.6 \\
\hline & $6-311+\mathrm{G}(3 \mathrm{df}, 2 \mathrm{pd})$ & -0.3 & 8.3 & -0.3 & 7.8 & -0.1 & 2.5 \\
\hline
\end{tabular}


TABLE III: Basis set incompleteness errors $\left(\mathrm{cm}^{-1}\right.$, relative to our reference values) in harmonic frequencies, fundamental frequencies, and anharmonic corrections for several basis sets used in conjunction with the B97-1 and B3LYP functionals.

\begin{tabular}{|c|c|c|c|c|c|c|c|}
\hline \multicolumn{2}{|r|}{ Property } & \multicolumn{2}{|c|}{ Harmonic Frequency } & \multicolumn{2}{|c|}{ Fundamental Frequency } & \multicolumn{2}{|c|}{ Correction } \\
\hline Functional & Basis set & mean & RMS & mean & RMS & mean & RMS \\
\hline \multirow[t]{17}{*}{ B97-1 } & $3-21 G$ & -38.2 & 134.5 & -32.4 & 127.2 & -5.6 & 26.8 \\
\hline & $6-31 G$ & -40.7 & 108.4 & -38.7 & 104.6 & -2.0 & 11.4 \\
\hline & $6-31 \mathrm{G}^{*}$ & 4.7 & 36.4 & 6.3 & 36.1 & -1.4 & 8.3 \\
\hline & cc-pVDZ & -17.3 & 44.0 & -15.5 & 41.7 & -1.7 & 8.0 \\
\hline & DZP & -7.8 & 29.6 & -5.4 & 29.7 & -2.2 & 7.5 \\
\hline & $6-31+\mathrm{G}^{* *}$ & -6.5 & 30.6 & -4.7 & 31.3 & -1.6 & 8.0 \\
\hline & pc-1 & -9.2 & 35.0 & -7.8 & 34.7 & -1.2 & 6.2 \\
\hline & $6-311+\mathrm{G}^{* *}$ & -13.7 & 38.7 & -10.8 & 36.3 & -2.7 & 9.1 \\
\hline & $6-311+\mathrm{G}(3 \mathrm{~d}, 2 \mathrm{p})$ & -11.3 & 34.0 & -8.3 & 32.6 & -2.8 & 8.3 \\
\hline & $\mathrm{TZ} 2 \mathrm{P}$ & -8.9 & 29.6 & -5.7 & 28.3 & -3.4 & 8.7 \\
\hline & cc-pVTZ & -9.7 & 34.1 & -6.5 & 31.2 & -3.2 & 8.2 \\
\hline & TZVPP & -9.7 & 32.4 & -7.5 & 31.1 & -2.0 & 8.2 \\
\hline & pc-2 & -8.2 & 31.5 & -5.8 & 30.2 & -2.3 & 7.0 \\
\hline & $6-311+\mathrm{G}(3 \mathrm{df}, 2 \mathrm{pd})$ & -8.9 & 33.1 & -5.8 & 31.5 & -2.9 & 7.7 \\
\hline & $\mathrm{TZV}(\mathrm{P}+1) \mathrm{P}$ & -8.3 & 31.8 & -6.0 & 30.4 & -2.1 & 8.2 \\
\hline & cc-pVQZ & -9.0 & 32.7 & -6.5 & 32.4 & -2.4 & 7.8 \\
\hline & pc-3 & -9.5 & 32.7 & -8.7 & 34.0 & -2.6 & 7.5 \\
\hline \multirow[t]{6}{*}{ B3LYP } & $6-31 \mathrm{G}^{*}$ & 3.1 & 41.3 & 3.6 & 41.3 & -0.3 & 6.5 \\
\hline & $6-31+\mathrm{G}^{* *}$ & -8.5 & 34.0 & -7.5 & 34.1 & -1.0 & 6.8 \\
\hline & $\mathrm{TZ} 2 \mathrm{P}$ & -7.6 & 31.5 & -5.2 & 30.4 & -2.3 & 6.7 \\
\hline & cc-pVTZ & -9.6 & 36.4 & -7.9 & 34.8 & -1.7 & 6.2 \\
\hline & $6-311+\mathrm{G}(3 \mathrm{df}, 2 \mathrm{pd})$ & -8.8 & 33.7 & -6.6 & 32.8 & -2.0 & 6.7 \\
\hline & pc-2 & -8.3 & 34.5 & -6.6 & 33.5 & -1.6 & 6.5 \\
\hline
\end{tabular}


TABLE IV: Errors $\left(\mathrm{cm}^{-1}\right)$ for equilibrium and vibrational ground state rotational constants (and difference between them) for several basis sets using the B97-1 and B3LYP functional, compared to the largest basis set used.

\begin{tabular}{|c|c|c|c|c|c|c|}
\hline \multicolumn{2}{|c|}{ Property } & \multicolumn{2}{|c|}{$B_{e}(\%)$} & \multicolumn{2}{|c|}{$B_{0}(\%)$} & Correction \\
\hline & Basis set & mean & RMS & mean & RMS & mean RMS \\
\hline \multicolumn{7}{|c|}{ B97-1 } \\
\hline minimal & $3-21 G$ & -6.13 & 8.66 & -6.26 & 8.76 & -0.0240 .070 \\
\hline \multirow[t]{6}{*}{ double-zeta } & $6-31 \mathrm{G}$ & -5.67 & 8.66 & -5.72 & 8.70 & -0.0110 .027 \\
\hline & $\mathrm{DZP}$ & -1.99 & 2.39 & -1.95 & 2.41 & -0.0030 .014 \\
\hline & cc-pVDZ & -1.87 & 2.33 & -1.80 & 2.35 & -0.0080 .032 \\
\hline & pc-1 & -1.84 & 2.25 & -1.80 & 2.27 & -0.0050 .014 \\
\hline & $6-31 G^{*}$ & -1.54 & 1.94 & -1.55 & 2.01 & -0.0120 .038 \\
\hline & $6-31+\mathrm{G}^{* *}$ & -1.43 & 1.78 & -1.41 & 1.80 & -0.0020 .006 \\
\hline \multirow[t]{8}{*}{ triple-zeta } & $6-311+\mathrm{G}^{* *}$ & -0.93 & 1.25 & -0.91 & 1.28 & $0.000 \quad 0.009$ \\
\hline & TZVPP & -0.47 & 1.00 & -0.48 & 1.04 & -0.0020 .085 \\
\hline & cc-pVTZ & -0.39 & 0.75 & -0.30 & 1.04 & -0.0020 .029 \\
\hline & pc-2 & -0.25 & 0.55 & -0.26 & 0.58 & -0.0010 .002 \\
\hline & $\mathrm{TZ2P}$ & -0.33 & 0.52 & -0.25 & 0.81 & $\begin{array}{ll}-0.005 & 0.027\end{array}$ \\
\hline & $6-311+\mathrm{G}(3 \mathrm{~d}, 2 \mathrm{p})$ & -0.24 & 0.34 & -0.18 & 0.35 & $0.000 \quad 0.005$ \\
\hline & $\mathrm{TZV}(\mathrm{P}+1) \mathrm{P}$ & -0.19 & 0.29 & -0.20 & 0.20 & -0.0020 .085 \\
\hline & $6-311+\mathrm{G}(3 \mathrm{df}, 2 \mathrm{pd})$ & -0.07 & 0.17 & -0.08 & 0.18 & $0.000 \quad 0.002$ \\
\hline quadruple-zeta & cc-pVQZ & -0.03 & 0.16 & -0.05 & 0.17 & -0.0010 .005 \\
\hline \multicolumn{7}{|c|}{ B3LYP } \\
\hline \multirow[t]{2}{*}{ double-zeta } & $6-31 \mathrm{G}^{*}$ & -1.40 & 1.82 & -1.40 & 1.90 & -0.0120 .042 \\
\hline & $6-31+\mathrm{G}^{* *}$ & -1.30 & 1.52 & -1.27 & 1.52 & -0.0010 .006 \\
\hline \multirow[t]{3}{*}{ triple-zeta } & cc-pVTZ & -0.15 & 0.63 & -0.07 & 0.93 & $0.002 \quad 0.029$ \\
\hline & $6-311+\mathrm{G}(3 \mathrm{df}, 2 \mathrm{pd})$ & 0.20 & 0.61 & 0.21 & 0.61 & $0.000 \quad 0.002$ \\
\hline & TZ2P & -0.05 & 0.60 & 0.12 & 0.48 & 0.0260 .094 \\
\hline
\end{tabular}


TABLE V: Errors $\left(\mathrm{cm}^{-1}\right)$ for equilibrium and vibrational ground state rotational constants (and difference between them) for several basis sets using the B3LYP and B97-1 functional, compared to our reference values.

\begin{tabular}{|c|c|c|c|c|c|c|c|}
\hline \multicolumn{2}{|c|}{ Property } & \multicolumn{2}{|c|}{$B_{e}(\%)$} & \multicolumn{2}{|c|}{$B_{0}(\%)$} & \multicolumn{2}{|c|}{ Correction } \\
\hline Functional & Basis set & mean & RMS & mean & RMS & mean & RMS \\
\hline \multirow[t]{17}{*}{ B97-1 } & $3-21 \mathrm{G}$ & -6.13 & 8.82 & -6.22 & 8.87 & -0.016 & 0.048 \\
\hline & $6-31 \mathrm{G}$ & -5.95 & 9.22 & -5.96 & 9.25 & -0.002 & 0.031 \\
\hline & $\mathrm{DZP}$ & -2.22 & 2.73 & -2.16 & 2.72 & 0.004 & 0.038 \\
\hline & cc-pVDZ & -2.08 & 2.56 & -2.03 & 2.72 & 0.000 & 0.032 \\
\hline & $6-31 \mathrm{G}^{*}$ & -1.75 & 2.21 & -1.72 & 2.24 & -0.003 & 0.034 \\
\hline & $6-31+\mathrm{G}^{* *}$ & -1.63 & 2.17 & -1.57 & 2.23 & 0.006 & 0.044 \\
\hline & pc-1 & -2.02 & 2.59 & -1.97 & 2.63 & 0.003 & 0.038 \\
\hline & $6-311+\mathrm{G}^{* *}$ & -1.16 & 1.71 & -1.57 & 1.80 & 0.008 & 0.052 \\
\hline & TZVPP & -0.67 & 1.45 & -0.60 & 1.58 & 0.006 & 0.045 \\
\hline & cc-pVTZ & -0.56 & 1.12 & -0.37 & 1.09 & 0.010 & 0.037 \\
\hline & pc-2 & -0.41 & 1.07 & -0.33 & 1.24 & 0.007 & 0.048 \\
\hline & TZ2P & -0.46 & 1.01 & -0.23 & 1.12 & 0.013 & 0.043 \\
\hline & $6-311+\mathrm{G}(3 \mathrm{~d}, 2 \mathrm{p})$ & -0.42 & 0.88 & -0.26 & 1.00 & 0.007 & 0.047 \\
\hline & $\mathrm{TZV}(\mathrm{P}+1) \mathrm{P}$ & -0.35 & 0.84 & -0.27 & 1.02 & 0.006 & 0.045 \\
\hline & $6-311+\mathrm{G}(3 \mathrm{df}, 2 \mathrm{pd})$ & -0.25 & 0.77 & -0.16 & 0.98 & 0.007 & 0.048 \\
\hline & cc-pVQZ & -0.19 & 0.75 & -0.12 & 0.95 & 0.006 & 0.045 \\
\hline & pc-3 & -0.16 & 0.74 & -0.07 & 0.97 & 0.007 & 0.048 \\
\hline \multirow[t]{6}{*}{ B3LYP } & $6-31 \mathrm{G}^{*}$ & -1.65 & 2.21 & -1.64 & 2.27 & -0.005 & 0.035 \\
\hline & $6-31+\mathrm{G}^{* *}$ & -1.55 & 2.24 & -1.50 & 2.32 & -0.005 & 0.045 \\
\hline & cc-pVTZ & -0.36 & 0.99 & -0.37 & 1.09 & 0.008 & 0.037 \\
\hline & $6-311+\mathrm{G}(3 \mathrm{df}, 2 \mathrm{pd})$ & -0.01 & 0.92 & 0.06 & 1.17 & 0.007 & 0.050 \\
\hline & TZ2P & -0.22 & 1.04 & -0.23 & 1.12 & 0.015 & 0.048 \\
\hline & pc-2 & -0.20 & 1.17 & -0.13 & 1.24 & 0.006 & 0.050 \\
\hline
\end{tabular}


TABLE VI: RMS errors using different basis sets for thermodynamic functions at several temperatures using DFT anharmonic force fields

\begin{tabular}{|c|c|c|c|c|c|c|c|c|c|c|}
\hline \multicolumn{2}{|c|}{ Property } & \multicolumn{3}{|c|}{ Heat capacity } & \multicolumn{3}{|c|}{ Enthalpy function } & \multicolumn{3}{|c|}{ Entropy } \\
\hline & & \multicolumn{3}{|c|}{$C_{p}[\mathrm{~J} / \mathrm{K} . \mathrm{mol}]$} & \multicolumn{3}{|c|}{$H-H_{0}[\mathrm{~kJ} / \mathrm{mol}]$} & \multicolumn{3}{|c|}{$S[\mathrm{~J} / \mathrm{K} . \mathrm{mol}]$} \\
\hline Functional & Basis set & 298.15 & 6002 & 2000 & 298.15 & 600 & 2000 & 298.15 & 600 & 2000 \\
\hline \multirow[t]{16}{*}{ B97-1 } & $3-21 G$ & 1.13 & 1.03 & 2.36 & 0.19 & 0.49 & 2.26 & 1.93 & 2.57 & 3.38 \\
\hline & $6-31 \mathrm{G}$ & 1.65 & 1.20 & 1.00 & 0.35 & 0.76 & 1.50 & 3.03 & 4.00 & 4.60 \\
\hline & $6-31 G^{*}$ & 0.29 & 0.36 & 0.65 & 0.06 & 0.14 & 0.62 & 0.55 & 0.67 & 0.86 \\
\hline & $6-31+\mathrm{G}^{* *}$ & 0.50 & 0.40 & 0.76 & 0.11 & 0.24 & 0.81 & 0.84 & 1.15 & 1.53 \\
\hline & $6-311+\mathrm{G}^{* *}$ & 0.50 & 0.40 & 0.94 & 0.10 & 0.24 & 0.84 & 0.75 & 1.06 & 1.53 \\
\hline & $\mathrm{DZP}$ & 0.56 & 0.40 & 0.72 & 0.12 & 0.26 & 0.70 & 0.96 & 1.28 & 1.54 \\
\hline & cc-pVDZ & 0.57 & 0.41 & 0.57 & 0.12 & 0.27 & 0.64 & 0.98 & 1.32 & 1.56 \\
\hline & pc-1 & 0.46 & 0.36 & 0.57 & 0.10 & 0.22 & 0.68 & 0.80 & 1.08 & 1.41 \\
\hline & TZVPP & 0.35 & 0.29 & 0.75 & 0.07 & 0.16 & 0.57 & 0.53 & 0.74 & 0.95 \\
\hline & cc-pVTZ & 0.33 & 0.30 & 0.69 & 0.06 & 0.15 & 0.55 & 0.37 & 0.56 & 0.79 \\
\hline & pc-2 & 0.28 & 0.23 & 0.73 & 0.05 & 0.12 & 0.45 & 0.34 & 0.50 & 0.64 \\
\hline & TZ2P & 0.31 & 0.29 & 0.55 & 0.04 & 0.14 & 0.56 & 0.35 & 0.57 & 0.81 \\
\hline & $6-311+\mathrm{G}(3 \mathrm{~d}, 2 \mathrm{p})$ & 0.42 & 0.38 & 0.80 & 0.07 & 0.19 & 0.68 & 0.45 & 0.73 & 1.09 \\
\hline & $6-311+\mathrm{G}(3 \mathrm{df}, 2 \mathrm{pd})$ & 0.36 & 0.32 & 0.72 & 0.06 & 0.17 & 0.58 & 0.45 & 0.69 & 0.96 \\
\hline & cc-pVQZ & 0.25 & 0.24 & 0.65 & 0.05 & 0.13 & 0.44 & 0.36 & 0.54 & 0.71 \\
\hline & pc-3 & 0.26 & 0.25 & 0.70 & 0.05 & 0.13 & 0.57 & 0.35 & 0.53 & 0.95 \\
\hline \multirow[t]{6}{*}{ B3LYP } & $6-31 \mathrm{G}^{*}$ & 0.34 & 0.39 & 0.51 & 0.06 & 0.16 & 0.47 & 0.54 & 0.69 & 0.81 \\
\hline & $6-31+\mathrm{G}^{* *}$ & 0.52 & 0.41 & 0.67 & 0.11 & 0.24 & 0.70 & 0.84 & 1.15 & 1.46 \\
\hline & cc-pVTZ & 0.33 & 0.30 & 0.66 & 0.06 & 0.15 & 0.49 & 0.37 & 0.95 & 1.07 \\
\hline & $6-311+\mathrm{G}(3 \mathrm{df}, 2 \mathrm{pd})$ & 0.39 & 0.35 & 0.78 & 0.06 & 0.18 & 0.63 & 0.45 & 0.70 & 1.03 \\
\hline & TZ2P & 0.34 & 0.34 & 0.92 & 0.05 & 0.15 & 0.76 & 0.42 & 0.59 & 0.93 \\
\hline & pc-2 & 0.32 & 0.28 & 0.78 & 0.05 & 0.14 & 0.49 & 0.36 & 0.56 & 0.76 \\
\hline
\end{tabular}


TABLE VII: Errors $\left(\mathrm{cm}^{-1}\right)$ in harmonic frequencies, fundamental frequencies, and anharmonic corrections for several methods.

\begin{tabular}{|cc|cc|ll|ll|}
\hline \hline \multicolumn{2}{|c|}{ Property } & Harmonic Frequency & Fundamental Frequency & Correction \\
\hline Type & Method & mean & RMS & mean & RMS & mean RMS \\
\hline GGA & BLYP & -61 & 107 & -60 & 107 & -0.3 & 6.1 \\
GGA & HCTH/407 & -27 & 54 & -26 & 55 & -1.3 & 6.7 \\
GGA & PBE & -55 & 91 & -53 & 91 & -2.5 & 9.2 \\
mGGA & TPSS & -29 & 61 & -26 & 62 & -2.8 & 11.1 \\
Hybrid(AE) & B3LYP & -5 & 34 & -2 & 34 & -2.5 & 7.5 \\
Hybrid(AE) & B97-1 & -7 & 32 & -3 & 32 & -4.0 & 10.3 \\
Hybrid(AE) & B97-2 & 9 & 33 & 11 & 37 & -2.6 & 6.6 \\
Hybrid(AE) & PBE0 & 8 & 34 & 12 & 39 & -3.8 & 9.1 \\
Hybrid(AE) & TPSSh & -7 & 35 & -4 & 35 & -2.7 & 8.6 \\
Hybrid(AE) & mPW1B95 & 20 & 43 & 22 & 51 & -2.1 & 12.8 \\
Hybrid(TS) & mPW1K & 52 & 89 & 56 & 97 & -4.2 & 9.8 \\
Hybrid(TS) & mPWB1K & 49 & 86 & 50 & 93 & -0.9 & 14.4 \\
Hybrid(TS) & BMK & 20 & 58 & 26 & 58 & -5.9 & 21.8 \\
ab initio & HF & 105 & 324 & 113 & 329 & -7.4 & 22.5 \\
ab initio & MP2 & 16 & 56 & 21 & 61 & -5.0 & 12.7 \\
ab initio & MP2/pVTZ & 16 & 45 & 22 & 53 & -5.1 & 12.8 \\
\hline \hline
\end{tabular}


TABLE VIII: Errors $\left(\mathrm{cm}^{-1}\right)$ for equilibrium and vibrational ground state rotational constants (and difference between them) for several exchange-correlation functionals.

\begin{tabular}{|c|c|c|c|c|c|c|}
\hline \multicolumn{2}{|c|}{ Property } & \multicolumn{2}{|c|}{$B_{e}(\%)$} & \multicolumn{2}{|c|}{$B_{0}(\%)$} & \multirow{2}{*}{\begin{tabular}{|l} 
Correction \\
mean RMS
\end{tabular}} \\
\hline Туре & Method & mean & RMS & mean & RMS & \\
\hline GGA & BLYP & -2.28 & 2.77 & -2.19 & 2.81 & 0.0140 .051 \\
\hline GGA & НCTH/407 & -0.61 & 1.25 & -0.50 & 1.30 & 0.0050 .040 \\
\hline GGA & PBE & -2.01 & 2.32 & -1.84 & 2.28 & $\begin{array}{lll}0.018 & 0.057\end{array}$ \\
\hline mGGA & TPSS & -1.28 & 1.83 & -1.19 & 1.92 & $\begin{array}{lll}0.001 & 0.039\end{array}$ \\
\hline Hybrid(AE) & B3LYP & -0.18 & 1.05 & 0.14 & 1.12 & 0.0150 .047 \\
\hline Hybrid(AE) & B97-1 & -0.42 & 1.01 & -0.19 & 1.03 & 0.0120 .043 \\
\hline Hybrid(AE) & B97-2 & 0.34 & 0.92 & 0.61 & 1.15 & $\begin{array}{lll}0.015 & 0.047\end{array}$ \\
\hline Hybrid(AE) & PBE0 & 0.25 & 0.90 & 0.59 & 1.19 & 0.0180 .051 \\
\hline Hybrid(AE) & TPSSh & -0.95 & 1.66 & -0.89 & 1.81 & $\begin{array}{lll}0.002 & 0.035\end{array}$ \\
\hline Hybrid(AE) & mPW1B95 & 0.90 & 1.30 & 1.10 & 1.64 & 0.0090 .064 \\
\hline Hybrid(TS) & $\mathrm{mPW} 1 \mathrm{~K}$ & 1.76 & 2.05 & 2.00 & 2.42 & 0.0180 .051 \\
\hline Hybrid(TS) & mPWB1K & 1.93 & 2.19 & 2.30 & 2.66 & 0.0180 .051 \\
\hline Hybrid(TS) & BMK & 0.34 & 1.28 & 0.57 & 1.56 & $\begin{array}{lll}0.017 & 0.065\end{array}$ \\
\hline ab initio & $\mathrm{HF}$ & 3.22 & 3.77 & 3.60 & 4.28 & 0.0220 .079 \\
\hline ab initio & MP2 & -0.05 & 1.35 & -0.02 & 1.46 & 0.0050 .042 \\
\hline ab initio & $\mathrm{MP} 2 / \mathrm{pVTZ}$ & 0.12 & 1.06 & 0.23 & 1.07 & 0.0130 .102 \\
\hline
\end{tabular}


TABLE IX: RMS errors for thermodynamic functions at several temperatures using DFT anharmonic force fields

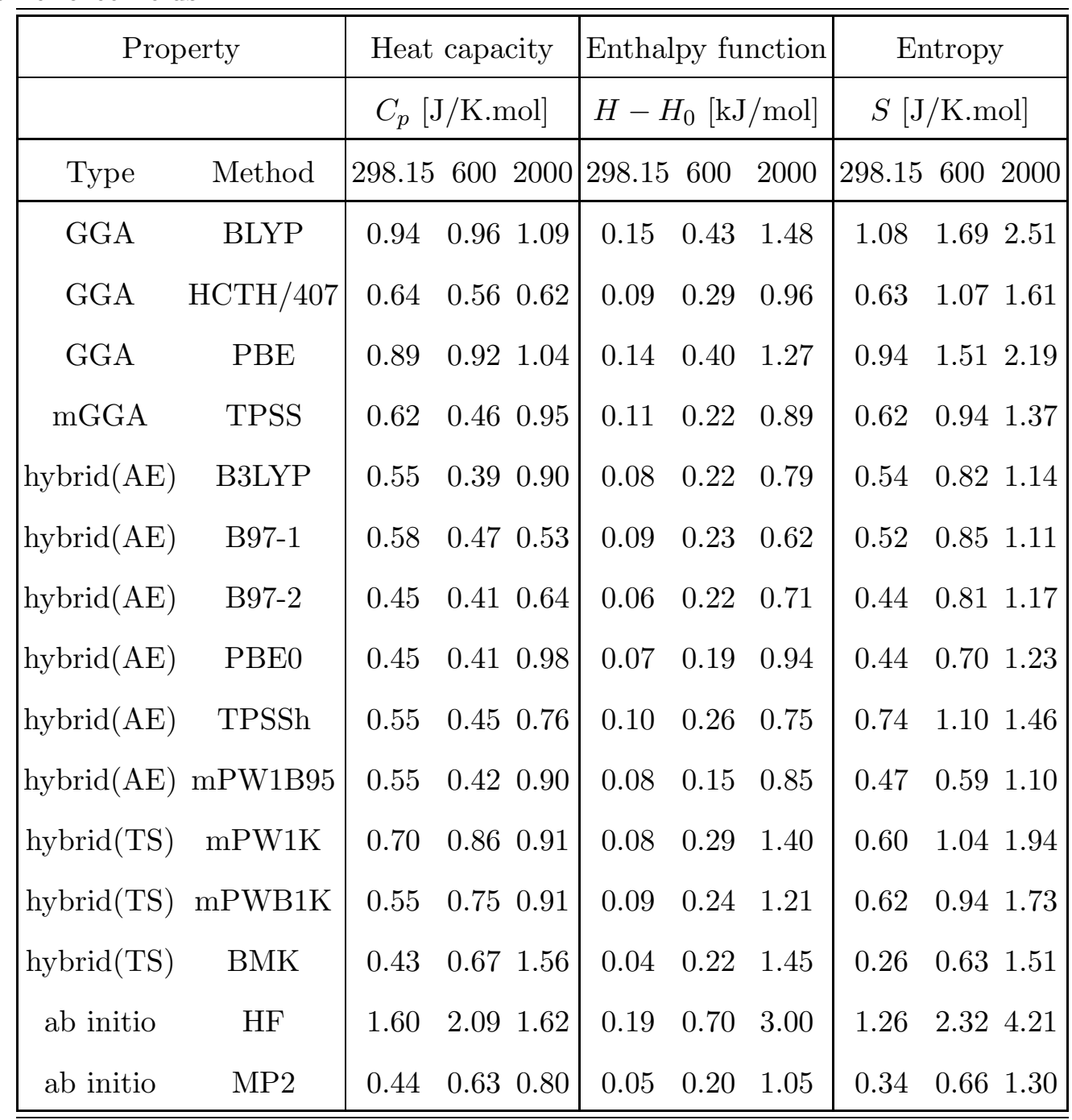

